\title{
Anticoagulation during Renal Replacement Therapy
}

\author{
Simran Singh \\ Keywords: Continuous renal replacement therapy, Low-molecular-weight heparin, Unfractionated heparin. \\ Indian Journal of Critical Care Medicine (2020): 10.5005/jp-journals-10071-23412
}

\section{INTRODUCTION}

Anticoagulation is generally required for acute renal replacement therapy (RRT).

Renal replacement therapies used for acute kidney injury include intermittent hemodialysis, slow low-efficiency daily dialysis, continuous RRT (CRRT), and peritoneal dialysis.

Most intensive care unit patients can avoid any anticoagulation during intermittent hemodialysis or sustained low efficiency daily dialysis (SLEDD) because of short treatment duration and higher flow rates. This may not be possible during CRRT as the contact of blood with the foreign surface of the extracorporeal circuit results in activation of coagulation pathways and platelets. Selecting an anticoagulant agent is determined by the patient's underlying illness, and not only the availability of the anticoagulant but also the technical expertise.

Anticoagulation reduces filter clotting and improves the efficacy of renal replacement therapy as well as prolongs the filter life. Replacing the hemofilter due to clotting of the circuit may reduce the time on CRRT from 24 to 16 hours a day, thereby reducing the delivery of an adequate CRRT dose. ${ }^{1}$

Circuit clotting is the most frequent cause of therapy interruption in CRRT. ${ }^{2}$

It also adds to increased cost of the therapy due to frequent hemofilter changes.

The ideal anticoagulant for CRRT should provide optimal antithrombotic activity with low bleeding risk and minimal systemic adverse effects. It should also have a short half-life and have an antidote for easy reversal.

This article will focus attention on anticoagulant strategies used to prevent clotting in the CRRT circuit.

\section{Anticoagulant Options during CRRT}

\begin{tabular}{l}
\hline Unfractionated Heparin \\
LMWH \\
Citrate \\
No anticoagulation \\
$\quad$ Saline flushes \\
Prostacyclin \\
Direct thrombin inhibitors \\
$\quad$ Hirudin, argatroban \\
Serine protease inhibitors \\
$\quad$ Nafamostat \\
\hline
\end{tabular}

Department of Intensive Care Medicine, P.D. Hinduja Hospital, Mahim, Mumbai, Maharashtra, India

Corresponding Author: Simran Singh, Department of Intensive Care Medicine, P.D. Hinduja Hospital, Mahim, Mumbai, Maharashtra, India, e-mail: simranjsingh@hotmail.com

How to cite this article: Singh S. Anticoagulation during Renal Replacement Therapy. Indian J Crit Care Med 2020;24(Suppl 3): S112-S116.

Source of support: Nil

Conflict of interest: None

\section{Systemic Anticoagulation with Heparin}

Unfractionated or standard heparin (UFH) is the anticoagulant agent most commonly used in CRRT to prolong the life of the extracorporeal circuit.

The UFH inactivates factors Xa and Ila. The half-life of UFH is 90 minutes; however, in renal failure it may be increased up to 3 hours. Advantages of UFH is that it is inexpensive, has a relatively short half-life, and is easily reversed with protamine.

Heparin is given as a continuous infusion at the arterial site of the circuit. Circuit priming with heparin added to normal saline helps the negatively charged heparin molecules to adhere to the circuit tubing. For priming the circuit, 5,000 to 10,000 units of heparin can be added to $1 \mathrm{~L}$ of $0.9 \%$ saline. A bolus of 1,000 to $5,000 \mathrm{IU}$ of heparin can also be given. Continuous infusion of heparin ranges between 5 and 20 units/kg/hour.

Bellomo and Ronco suggest a dose of $8-10$ units $/ \mathrm{kg} / \mathrm{hour}$ be administered into the blood line before the hemofilter, especially for those patients who are at low risk of bleeding. ${ }^{3}$

The anticoagulant effect of heparin is monitored by measuring the activated partial thromboplastin time (aPTT). Aim is to target aPTT between 1.5 and 2.0 times the normal.

\section{Heparin-protamine Anticoagulation}

Regional heparin-protamine anticoagulation is administered by infusing UFH into the arterial line of the extracorporeal circuit followed by post-filter infusion of protamine. The dose of protamine must be effective to bind the pre-filter-infused UFH. This requires measurement of aPTT in the circuit and systemically. The circuit aPTT should be doubled, while the systemic aPTT should be within the normal range. ${ }^{4}$

The heparin-protamine complexes after being taken up by the reticuloendothelial system are released into the circulation. The elimination half-lives of heparin and protamine differ significantly, therefore, calculating an accurate dose can be challenging. ${ }^{5}$

Also protamine can exert serious adverse effects, including hypotension, release of complement factors, histamine, and

() The Author(s). 2020 Open Access This article is distributed under the terms of the Creative Commons Attribution 4.0 International License (https://creativecommons. org/licenses/by-nc/4.0/), which permits unrestricted use, distribution, and non-commercial reproduction in any medium, provided you give appropriate credit to the original author(s) and the source, provide a link to the Creative Commons license, and indicate if changes were made. The Creative Commons Public Domain Dedication waiver (http://creativecommons.org/publicdomain/zero/1.0/) applies to the data made available in this article, unless otherwise stated. 
other inflammatory mediators. Protamine may cause pulmonary hypertension and right heart failure. It may also impair coagulation. ${ }^{6}$

Therefore, it is fair to say that regional heparin-protamine anticoagulation is a complex intervention with a high risk of adverse effects. It is not superior to other forms of anticoagulation. Hence, it cannot be recommended for clinical practice. ${ }^{7}$

\section{Complications Associated with Heparin}

Disadvantages of heparin include systemic bleeding, unpredictable kinetics - aPTT being an unreliable predictor of bleeding, heparin resistance, and heparin-induced thrombocytopenia (HIT). ${ }^{8}$

\section{Heparin-induced Thrombocytopenia}

Heparin-induced thrombocytopenia is an adverse effect of systemic heparin therapy. Binding of heparin complex and platelet factor 4 (PF4) can trigger antibody production. In medical and surgical patients, the occurrence of this antibody production varies between $8 \%$ and $17 \% .{ }^{9}$

These antibodies also cause platelet activation, leading to a prothrombotic state, which can result in a potentially lifethreatening thrombosis or thromboembolism.

\section{Heparin Resistance}

There is a large variability of heparin response among patients. The term "heparin resistance" is used when a total dose of more than 35,000 IU of intravenous UFH per day is required to prolong the aPTT 1.5 to 2 times from the normal. ${ }^{10}$

Heparin resistance manifests in the presence of antithrombin (AT) III deficiency. While congenital AT III deficiency is uncommon, many clinical conditions (e.g., perioperative bleeding, shock, liver cirrhosis, hemodialysis itself) can lead to low levels of AT III, resulting in an inefficient anticoagulation by heparin which in turn results in a reduced filter life span.

Also AT III has anti-inflammatory effects. By heparin binding, this protective effect of AT III gets diminished. ${ }^{10}$

Heparin resistance can also develop despite normal AT III levels especially in cardiac surgery patients as they might have elevated levels of factor VIII. Heparin binding to factor VIII can also contribute to the development of heparin resistance. ${ }^{11}$

In view of the above complication with heparin, the kidney diseases: improving global outcome (KDIGO) guidelines suggest using regional citrate anticoagulation (RCA) in those without contraindications to citrate. However, administration of UFH as an anticoagulant still remains the most preferred choice during CRRT. ${ }^{12}$

\section{Unfractionated Heparin vs Low-molecular-weight Heparin}

Unfractionated heparin or low-molecular-weight heparin (LMWH) is used for patients with a low risk of bleeding and those who have an absolute contraindication to citrate administration.

The LMWH is expensive, has a prolonged life, and has not been found to be superior to UFH.

A main concern of using $\mathrm{LMWH}$ is systemic anticoagulation and the lack of a reliable predictor of bleeding and antithrombotic efficacy.

Disadvantages of LMWH are increased half-life compared to UFH and poor reversibility with protamine and costs. Daily costs of LMWH including the anti-Xa assays are $10 \%$ higher than UFH.

Studies have compared fixed-dose LMWH with UFH and found no distinct advantage in circuit survival. Meta-analyses and the American College of Chest Physicians guidelines conclude that the use of LMWH is associated with major bleeding in patients with a creatinine clearance of less than $30 \mathrm{~mL} /$ minute and recommend either UFH or a reduction in LMWH dose by $50 \%$ for such patients.

So far evidence does not support a recommendation of LMWH over UFH in the critically ill with acute kidney injury. ${ }^{13}$

\section{Thrombin Antagonists}

Direct thrombin inhibitors (e.g., argatroban), AT-dependent factor Xa inhibitors (e.g., fondaparinux), and serine protease inhibitors (nafamostat mesilate) are the other anticoagulants though not frequently used as most centers lack experience.

Argatroban does not cross-react with heparin antibodies and is the preferred approach for HIT because of its hepatic clearance (half-life $\sim 35$ minutes). It is administered as a $0.1-0.25 \mathrm{mg} / \mathrm{kg}$ bolus before intermittent hemodialysis or an infusion of $0.1-0.2 \mathrm{mg} / \mathrm{kg} /$ hour during CRRT and titrated per activated partial thromboplastin time (APPT). Bivalirudin is used as an alternative to argatroban in patients who have combined liver and renal failure because of its extrarenal and extrahepatic clearance. It has a short half-life with reversible thrombin binding ${ }^{14}$ (Table 1).

\section{Heparinoids}

Danaparoid is a heparinoid derived from pig intestinal mucosa. It has a high anti-Xa and low anti-lla effect. Although danaparoid has been used in HIT, it has a cross-reactivity with heparin/PF4 and antibodies are reported in $5-10 \%$ of patients. Also, in renal failure its half-life is prolonged (36-48 hours). Danaparoid has no antagonist.

Despite a considerably lower mean anti-Xa activity $(0.4 \pm 0.2$ anti-Xa U/mL), bleeding occurred in $46 \%$ of the patients.

\section{Citrate Anticoagulation}

Citrate is an anticoagulant and a buffer. Sodium citrate administered pre-filter results in chelation of calcium. This results in regional hypocalcemia in the filter and as a result there will be inhibition of thrombin generation. Citrate has a plasma half-life of 5 minutes and is metabolized primarily in the liver to bicarbonate. It is partially removed by convection or diffusion as complexed calcium citrate and the remaining amount is rapidly metabolized in the citric acid (Krebs) cycle - especially in the liver, muscle, and renal cortex.

The chelated calcium is released and the lost calcium is replaced. The extracorporeal clearance of citrate is the same as that of urea (sieving coefficient 0.87-1.0).

Post-filter ionized calcium ( $\mathrm{iCa}$ ) is monitored and used to titrate the citrate rate to ensure anticoagulation. The goal is to maintain the iCa at less than $0.40 \mathrm{mmol} / \mathrm{L}$ which is essential to inhibit the coagulation pathway. The returning blood combines with the venous blood in the body, which normalizes the iCa and prevents systemic anticoagulation.

This means that the patient loses some of their calcium. Calcium must therefore be given back to the patient via a central line. Calcium-free dialysate solution should be used, as no calcium should be present in the circuit.

The commercially prepared citrate formulations for CRRT are not available universally. These citrate solutions used for RCA are either custom-made or available in a concentrated formulation with high sodium content that are not safe for CRRT use. These hypertonic citrate formulations often require compensatory hyponatremic replacement or dialysate solutions with either nil or reduced bicarbonate concentrations to prevent the development of metabolic alkalosis. The most commonly used hypertonic citrate formulations include $2.2 \%$ anticoagulant citrate dextrose solution, which contains $224 \mathrm{mmol} / \mathrm{L}$ sodium, $74.8 \mathrm{mmol} / \mathrm{L}$ citrate, and 
Table 1: Dosing recommendations for anticoagulation during continuous renal replacement therapy

\begin{tabular}{lllll}
\hline & Loading & Maintenance & Monitoring & Target \\
\hline Unfractionated heparin & $2000-5000 \mathrm{U}$ & $5-10 \mathrm{IU} / \mathrm{kg} / \mathrm{hour}$ & $\mathrm{aPTT}$ & $1-1.4$ times normal \\
Dalteparin & $15-25 \mathrm{IU} / \mathrm{kg}$ & $5 \mathrm{IU} / \mathrm{kg} / \mathrm{hour}$ & Anti-Xa & $0.25-0.35 \mathrm{IU} / \mathrm{mL}$ \\
Enoxaparin & $0.15 \mathrm{mg} / \mathrm{kg}$ & $0.05 \mathrm{mg} / \mathrm{kg} / \mathrm{hour}$ & Anti-Xa & $0.25-0.35 \mathrm{IU} / \mathrm{mL}$ \\
Danaparoid & $750 \mathrm{U}$ & $1-2 \mathrm{U} / \mathrm{kg} / \mathrm{hour}$ & Anti-Xa & $0.25-0.35 \mathrm{IU} / \mathrm{mL}$ \\
Fondaparinux & No loading & $2.5 \mathrm{mg} / \mathrm{day}$ & Anti-Xa & $0.25-0.35 \mathrm{IU} / \mathrm{mL}$ \\
Argatroban & $250 \mu \mathrm{g} / \mathrm{kg}$ & $0.5-2 \mu \mathrm{g} / \mathrm{kg} / \mathrm{minute}$ & $\mathrm{aPTT}$ & $1-1.4$ times normal \\
R-Hirudin & No loading & $0.005-0.01 \mathrm{mg} / \mathrm{kg} / \mathrm{hour}$ and after $1-2$ days 0.005 & ECT & $80-100$ seconds \\
& & $\mathrm{mg} / \mathrm{kg} / \mathrm{hour}$ or intermittent bolus $0.002 \mathrm{~g} / \mathrm{kg}$ & & Prefilter aPTT > \\
Nafamostat & No loading & $0.1-0.5 \mathrm{mg} / \mathrm{kg} / \mathrm{hour}$ & aPTT & $2-2.5$ times normal \\
\hline
\end{tabular}

aPTT, activated partial thromboplastin time; ECT, ecarin clotting time

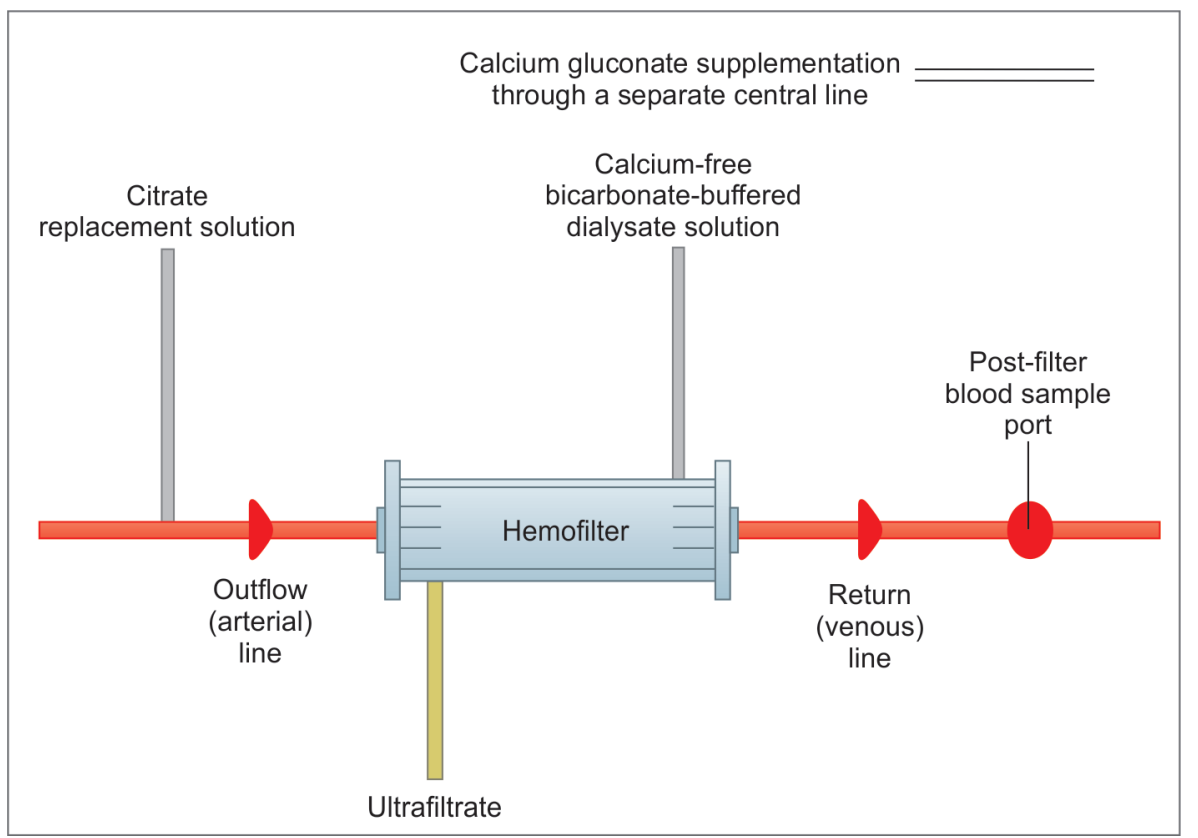

Fig. 1: Regional citrate anticoagulation for continuous venovenous hemodiafiltration

$38 \mathrm{mmol} / \mathrm{L}$ citric acid and 4\% trisodium citrate solution, which contains $420 \mathrm{mmol} / \mathrm{L}$ sodium and $136 \mathrm{mmol} / \mathrm{L}$ citrate.

Two isotonic dilute citrate formulations specifically for CRRT have become commercially available. These isotonic solutions are meant to function as a combined anticoagulant and replacement fluid for convective clearance in continuous venovenous hemofiltration (CVVH) and continuous venovenous hemodiafiltration (CVVHDF). ${ }^{15}$

An iCa concentration below $0.35 \mathrm{mmol} / \mathrm{L}$ is required to inhibit coagulation. ${ }^{16}$

\section{Metabolic Complications Associated with Citrate Anticoagulation}

Complications include metabolic alkalosis, metabolic acidosis, hypocalcemia, hypernatremia from hyperosmolar citrate solutions (4\% sodium citrate), and hypercalcemia from inappropriate calcium supplementation. The risk factors for citrate toxicity include fulminant hepatic failure or shock liver and nursing errors leading to overdose.

Citrate toxicity can be suspected when there is evidence of a rising anion gap, worsening metabolic acidosis, a falling systemic $\mathrm{iCa}$, escalating calcium infusion requirements, or a total calciumsystemic iCa ratio greater than 2.5:1.
Citrate toxicity can be minimized by decreasing the citrate infusion rate, decreasing the blood pump speed, and increasing the dialysate flow rate.

Circuit survival with citrate is favorable as compared to UFH. The major advantage with citrate being that adequate circuit anticoagulation can be obtained without systemic anticoagulation, thereby minimizing the risk of bleeding.

Analyzing data from the Program to Improve Care in Acute Renal Disease study, Claure-del Granado and colleagues evaluated the association of an anticoagulation strategy and effective solute clearance and circuit life. The investigators showed that the use of regional citrate for anticoagulation in CRRT significantly prolonged filter life and increased its efficacy in terms of delivered dose. ${ }^{17}$

Anticoagulation with citrate is associated with a lower activation of coagulation and leukocytes than heparins, suggesting improved biocompatibility. ${ }^{18}$

Current evidence shows that anticoagulation with citrate is safe if liver function is adequate and if CRRT is guided by a strict protocol. Monitoring of electrolytes, $\mathrm{iCa}$, and acid-base is mandatory. Numerous trials have shown citrate yields no additional bleeding risk and leads to longer filter life compared with UFH (Fig. 1).,19 


\section{Advantages of Citrate}

Safe to use on patients with active bleeding, recent bleeding, or who are at risk of bleeding, e.g., recent surgery.

Safe to use with patients at risk of HIT.

Citrate acts as a buffer and is metabolized into three molecules of bicarbonates. This may be beneficial in cases of severe metabolic acidosis.

\section{Disadvantages of Citrate}

In patients with severe liver disease, citrate metabolism may be inadequate, resulting in citrate accumulation, metabolic acidosis, and hypocalcemia.

Close monitoring of $\mathrm{Ca}, \mathrm{pH}, \mathrm{HCO}_{3}$, and electrolytes is required.

\section{No Anticoagulation}

The option of no anticoagulation is used in patients with coagulopathies, hepatic failure, or thrombocytopenia. Circuits are primed with saline solution or heparin. Intermittent saline solution flushes may be used as well. The rates of filter clotting using this method vary widely; however, the mean filter life with no anticoagulation is generally suboptimal between 12 hours and 48 hours.

Bellomo and Ronco suggest no anticoagulation for patients who have a platelet count $<50,000$, an international normalized ratio $>2.0$, an aPTT $>60$ seconds or who are actively bleeding or have had a hemorrhagic event in the previous 24 hours. $^{3}$

\section{Nonanticoagulant Measures to Reduce Filter Clotting}

\section{Venous Access and Circuit}

The venous access, the hemofilter, and the venous air trap are the most common sites in the circuit for potential thrombosis. Catheter malfunction leads to intermittent stasis of blood flow, which promotes clotting and subsequent circuit failure.

Reduction in blood flow has been shown to contribute to circuit failure. To minimize blood flow interruptions, the use of a large-bore double-lumen central venous catheter is recommended. Access being either the right jugular vein or the femoral vein. The length of femoral catheters is preferably $20-24 \mathrm{~cm}$ and possibly $30 \mathrm{~cm}$ in adults, whereas internal jugular catheters are best placed with their tip in the right atrium. The subclavian position is discouraged, given the high risk of kinking, the potential of subclavian stenosis, and difficulties with future arteriovenous fistulas. Catheters with side holes should not be used, because turbulent flow initiates clotting.

To reduce thrombogenicity of the catheter membrane, surface coating with substances such as heparin or polyethylenimine has been applied. The use of polyethylenimine-coated membranes has not shown to prolong circuit life during $\mathrm{CVVH}$ without anticoagulation in the critically ill population.

Air traps allow for a relatively slow-moving column of blood to have constant contact with air, potentiating the formation of clots. At times a slightly longer circuit life can be achieved by raising the blood level in the air trap higher than the blood inlet port.

\section{Predilution vs Postdilution}

The use of predilution is another way to reduce hemoconcentration during hemofiltration. During predilution, the fluid lost by ultrafiltration is replaced before the filter rather than after the filter (postdilution). The predilution fluid dilutes the blood in the filter and thereby prevents hemoconcentration and may improve hemofilter life.

\section{Conclusion}

The CRRT most often than not requires anticoagulation to maintain filter patency, thereby ensuring the delivery of an adequate dialysis dose, and patient's underlying medical condition should be taken into consideration prior to deciding the anticoagulation strategy.

Different systemic anticoagulants are available, including heparin anticoagulation. All these are generally associated with significant adverse effect, especially bleeding complications.

Regional citrate anticoagulation is superior to systemic anticoagulation because it prolongs filter life and reduces bleeding complications.

Adverse effects of regional citrate anticoagulation are citrate accumulation and hypo-/hypercalcemia.

Protocol-driven monitoring often allows the continuation of RCA with a reduced citrate dose. Patients at risk of adverse effects are those with liver failure, hypoxemia, severe lactic acidosis, and shock. In hypoxemic patients, especially with acute respiratory distress syndrome, there is a risk of citrate accumulation and RCA might not be well tolerated.

Regional citrate anticoagulation can be recommended as the therapy of choice for the majority of critically ill patients require CRRT. This choice is also influenced by local expertise, nursing comfort, ease of monitoring, and availability of fluids. Although $\mathrm{UFH}$ is most commonly used, citrate anticoagulation is gaining wider acceptance as an alternative anticoagulation approach with the implementation of simplified protocols.

\section{References}

1. Venkataraman R, Kellum JA, Pavelsky P. Dosing pattern for continuous renal replacement at a large academic centre in United states. J Crit Care 2002;17(4):246-250. DOI: 10.1053/jcrc.2002.36757.

2. Joannidis M, Oudemans-van Straaten HM. Clinical review: Patency of the circuit in continuous renal replacement therapy. Crit Care 2007;11(4):218. DOI: 10.1186/cc5937.

3. Bellomo R, Ronco C. Anticoagulation during CRRT. In: Bellomo R, Baldwin I, Ronco C, Golper T. Atlas of haemofiltration. Sydney: W.B. Saunders; 2002. pp. 63-68.

4. Tolwani AJ, Wille KM. Anticoagulation for continuous renal replacement therapy. Semin Dial 2009;22(2):141e5. DOI: 10.1111/j.1525139X.2008.00545.x.

5. Butterworth J, Lin YA, Prielipp R, Bennett J, James R. The pharmacokinetics and cardiovascular effects of a single intravenous dose of protamine in normal volunteers. Anesth Analg 2002;94(3):514522. DOI: 10.1097/00000539-200203000-00008.

6. Carr JA, Silverman N. The heparin-protamine interaction. A review. The J Cardiovasc Surg 1999;40(5):659-666.

7. Gattas DJ, Rajbhandari D, Bradford C, Buhr H, Lo S, Bellomo R. A randomized controlled trial of regional citrate versus regional heparin anticoagulation for continuous renal replacement therapy in critically ill adults. Crit Care Med 2015;43(8):1622-1629. DOI: 10.1097/ CCM.0000000000001004.

8. Davenport A. Anticoagulation for continuous renal replacement therapy. Contribut Nephrol 2004;144:228-238. DOI: 10.1159/000078891.

9. Amiral J, Peynaud-Debayle E, Wolf M, Bridey F, Vissac AM, Meyer D. Generation of antibodies to heparin-PF4 complexes without thrombocytopenia in patients treated with unfractionated or lowmolecular-weight heparin. Am J Hematol 1996;52(2):90-95. DOI: 10.1002/(SICI)1096-8652(199606)52:23.0.CO;2-0. 
10. Leithäuser B, Schumacher J, Lendemans S, Tillmanns $H$, Matthias FR. Antithrombin attenuates microvascular leakage and leukocyteendothelial interaction in response to endotoxin. Semin Thromb Hemost 2002;28(Suppl. 1):87-94. DOI: 10.1055/s-2002-30201.

11. Thota R, Ganti AK, Subbiah S. Apparent heparin resistance in a patient with infective endocarditis secondary to elevated factor VIII levels. J Thromb Thrombolysis 2012;34(1):132-134. DOI: 10.1007/s11239012-0692-z.

12. Kellum JA, Lameire N, Aspelin P. Kidney disease: Improving global outcomes (KDIGO) acute kidney injury work group. KDIGO clinical practice guideline for acute kidney injury. Kidney Int Suppl 2012;2(1):1-138. DOI: 10.1038/kisup.2012.1.

13. Hirsh J, Bauer KA, Donati MB, Gould M, Samama MM, Weitz J. Parenteral anticoagulants: American College of Chest Physicians evidence-based clinical practice guidelines. 8 th ed. Chest 2008;133(Suppl. 6):141S-159S. DOI: 10.1378/chest.08-0689.

14. Murray PT, Reddy BV, Grossman EJ, Hammes MS, Trevino S, Ferrell J, et al. A prospective comparison of three argatroban treatment regimens during hemodialysis in end-stage renal disease. Kidney Int 2004;66(6):2446-2453. DOI: 10.1111/j.1523-1755.2004.66022.x.
15. Oudemans-Van Straaten HM, Bosman RJ, Koopmans M, van der Voort PH, Wester JP, van der Spoel Jl, et al. Citrate anticoagulation for continuous venovenous hemofiltration. Crit Care Med 2009;37(2):545-552. DOI: 10.1097/CCM.0b013e3181953c5e.

16. Oudemans-Van Straaten HM. Anticoagulation for renal replacement therapy: Heparin or citrate. In: Kuhlen R, Moreno R, Rhodes RM. Controversies in Intensive Care Medicine 2008. pp. 153-161.

17. Claure-Del Granado R, Macedo E, Soroko S, Kim Y, Chertow GM, Himmelfarb J, , et al. Anticoagulation, delivered dose and outcomes in CRRT: The program to improve care in acute renal disease (PICARD). Hemodial Int 2014;18(3):641-649. DOI: 10.1111/hdi.12157.

18. Hofbauer R, Moser D, Frass M, Oberbauer R, Kaye AD, Wagner O, et al. Effect of anticoagulation on blood membrane interactions during hemodialysis. Kidney Int 1999;56(4):1578-1583. DOI: 10.1046/j.15231755.1999.00671.x.

19. Bai M, Zhou M, He L, Ma F, Li Y, Yu Y, et al. Citrate versus heparin anticoagulation for continuous renal replacement therapy: an updated meta-analysis of RCTs. Intensive Care Med 2015;41(12):20982110. DOI: 10.1007/s00134-015-4099-0. 